\title{
Impaired context reversal learning, but not cue reversal learning, in patients with amnestic mild cognitive impairment
}

\author{
Einat Levy-Gigi a,*, Oguz Kelemen ${ }^{\mathrm{b}}$, Mark A. Gluck ${ }^{\mathrm{a}}$, Szabolcs Kéri ${ }^{\mathrm{c}, \mathrm{d}}$ \\ a Center for Molecular and Behavioral Neuroscience, Rutgers University, Newark, USA \\ ${ }^{\mathrm{b}}$ Bács-Kiskun County Hospital, Kecskemét, Hungary \\ ' Department of Physiology, Faculty of Medicine, University of Szeged, Szeged, Hungary \\ d National Psychiatry Center, Budapest, Hungary
}

\section{A R T I C L E I N F O}

\section{Article history:}

Received 10 June 2011

Received in revised form 3 August 2011

Accepted 7 August 2011

Available online 11 August 2011

\section{Keywords:}

Reversal learning

Context

Amnestic mild cognitive impairment

Medial temporal lobe

Hippocampus

Prefrontal cortex

\begin{abstract}
A B S T R A C T
It has been proposed that reversal learning is impaired following damage to the orbitofrontal and ventromedial frontal cortex (OFC/VMFC) and to the medial temporal lobe (MTL), including the hippocampal formation. However, the exact characteristics of the MTL-associated reversal learning deficit are not known. To investigate this issue, we assessed 30 newly diagnosed patients with amnestic mild cognitive impairment (aMCI) and 30 matched healthy controls. All patients fulfilled the aMCI criteria of the Mayo Clinic Alzheimer's Disease Research Center and underwent head magnetic resonance imaging that confirmed MTL atrophy. Reversal learning was assessed using a novel reinforcement learning task. Participants first acquired and then reversed stimulus-outcome associations based on negative and positive feedback (losing and gaining points). Stimuli consisted of a cue (geometric shapes) and a spatial context (background color or pattern). Neuropsychological assessment included tasks related to the MTL (paired associates learning), dorsolateral prefrontal cortex (DLPFC) (extradimensional shift, One-touch Stockings of Cambridge), and OFC/VMFC (Holiday Apartment Task). Results revealed that, relative to controls, patients with aMCI exhibited a marked reversal learning deficit, which was highly selective for the reversal of context. The acquisition of stimulus-outcome associations and cue reversal learning were spared. Performance on the context reversal learning task significantly correlated with the right hippocampal volume. In addition, patients with aMCI had deficits on tests related to DLPFC but not to OFC/VMFC. However, DLPFC dysfunctions were not associated with context reversal learning. These results suggest that MTL deficits in aMCI selectively affect context reversal learning when OFC/VMFC functions are spared. This deficit is not influenced by the valence of the outcome (positive or negative feedback) and by executive dysfunctions.
\end{abstract}

(c) 2011 Elsevier Ltd. All rights reserved.

\section{Introduction}

Adapting to environmental changes is one of the most fundamental challenges for every organism. Conditions that once were rewarding may become disadvantageous and non-adaptive and vice versa. Cognitive flexibility, including attentional set-shifting and reversal learning, is a crucial element of adaptation (Frank \& Claus, 2006; Robbins \& Arnsten, 2009). Clinical studies and animal models have shown that frontal lobe lesions cause marked cognitive rigidity (Chudasama \& Robbins, 2006). Specifically, lesion to the dorsolateral prefrontal cortex (DLPFC) and its rodent analogues results in deficits on tasks requiring the shifting of attentional sets,

\footnotetext{
* Corresponding author at: Center for Molecular and Behavioral Neuroscience, Rutgers University, 197 University Ave, Newark 07102, NJ, USA. Tel.: +1 973353 3669; fax: +1 9733531272 .

E-mail address: levygigie@gmail.com (E. Levy-Gigi).
}

whereas damage to the orbitofrontal and ventromedial frontal cortex (OFC/VMFC) is associated with reversal learning impairment (Birrell \& Brown, 2000; Dias, Robbins, \& Roberts, 1996). Additional brain regions implicated in reversal learning are the cortico-striatal system and the medial temporal lobe (MTL), including the hippocampal formation (e.g., Cools, Clark, Owen, \& Robbins, 2002; Marston, Everitt, \& Robbins, 1993; Myers, Deluca, Hopkins, \& Gluck, 2006; Shohamy, Myers, Hopkins, Sage, \& Gluck, 2009; Swainson et al., 2000). However, the potential difference between the roles of these brain regions is not fully understood.

During discrimination learning, individuals form an attentional set of stimulus dimensions relevant for responding (e.g., discrimination of stimuli according to their shape). In attentional set-shifting tasks, individuals must change the attentional set to efficiently respond to newly relevant stimulus dimensions (e.g., shifting from shape to color). In reversal learning, participants first acquire a stimulus discrimination rule, and then learn to reverse this choice without a change in the relevant stimulus dimension 
(Chudasama \& Robbins, 2006). Although this model explains a wide range of behavioral phenomena, current discrimination learning paradigms do not take into consideration that stimulus dimensions regularly occur in a specific context (Vakil, Raz, \& Levy, 2007). For example a central stimulus dimension (e.g., a shape) can be presented against a peripheral context (e.g., a color or a texture background). In this case, the central dimension, or cue, refers to the "what" information, whereas the peripheral background refers to the "where" information (Eichenbaum, Yonelinas, \& Ranganath, 2007).

In discrimination and reversal learning, both cue and context may be relevant (Wickens, 1987). Cue and context may serve as conditioned stimuli predicting the probability of reinforcement (Good \& Honey, 1991). Context can also serve as an occasion setter, indicating whether a specific cue will be reinforced or not (Penick \& Solomon, 1991). Therefore, a cue can be reinforced in a distinctive context but not in another one (Bouton, 1993). While the MTL is not necessary to mediate each type of contextual conditional learning, it is critical for contextual occasion setting (Bouton, 1993; Eichenbaum et al., 2007; Gluck \& Myers, 2001; Myers \& Gluck, 1994; Mayes, Macdonald, Donlan, Pears, \& Meudell, 1992).

The current study was designed to investigate the relationship between PFC and MTL functioning and cue and context reversal learning. We developed a novel task comparing reversal learning for cue and context. Fig. 1 depicts stimuli with two dimensions, a central shape (cue) embedded in a color/pattern context. The first phase of the task includes a discrimination learning procedure in which cards consisting of a cue and a context predict a specific outcome (reward or punishment, i.e. winning or losing points). In the subsequent reversal phase, there are two possibilities: shared cue the cue is unchanged but appears in a new context; shared context - a new cue is presented in the original context. The new cards are associated with the opposite outcome relative to the discrimination learning phase. Therefore, if the original card predicts reward, then the new card will predict punishment and vice versa. Participants must reverse the original discrimination rule in order to adapt to the new condition (Fig. 1 and Table 1 ).

Table 1

Phases of the cue and context reversal task.

\begin{tabular}{|c|c|}
\hline $\begin{array}{c}\text { Phase 1: } \\
\text { acquisition }\end{array}$ & $\begin{array}{c}\text { Phase 2: } \\
\text { retention and reversal }\end{array}$ \\
\hline $\mathrm{A}(1) \Rightarrow+$ & $\begin{array}{l}\mathrm{A}(1) \Rightarrow+ \\
\mathrm{E}(1) \Rightarrow- \\
\mathrm{A}(5) \Rightarrow-\end{array}$ \\
\hline $\mathrm{B}(2) \Rightarrow+$ & $\begin{array}{l}\mathrm{B}(2) \Rightarrow+ \\
\mathrm{F}(2) \Rightarrow- \\
\mathrm{B}(6) \Rightarrow-\end{array}$ \\
\hline$C(3) \Rightarrow-$ & $\begin{array}{l}C(3) \Rightarrow- \\
F(3) \Rightarrow+ \\
C(7) \Rightarrow+\end{array}$ \\
\hline $\mathrm{D}(4) \Rightarrow-$ & $\begin{array}{l}\mathrm{D}(4) \Rightarrow- \\
\mathrm{G}(4) \Rightarrow+ \\
\mathrm{D}(8) \Rightarrow+\end{array}$ \\
\hline
\end{tabular}

A-G, eight cue shapes; $1-8$, eight contexts (colors and patterns). Green refers to positive outcome $(+)$, red refers to negative outcome $(-)$
A Version 1: black cues and color context

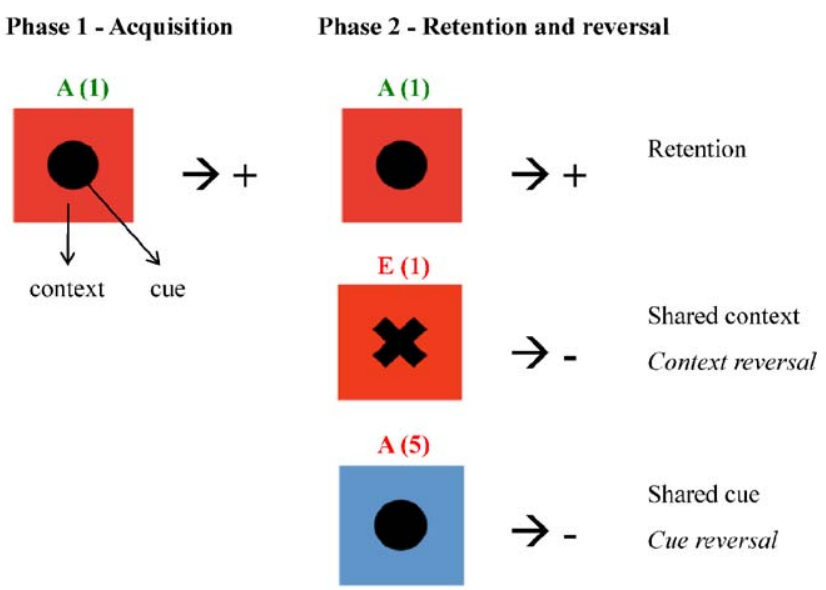

B Version 2: color cue with pattern context

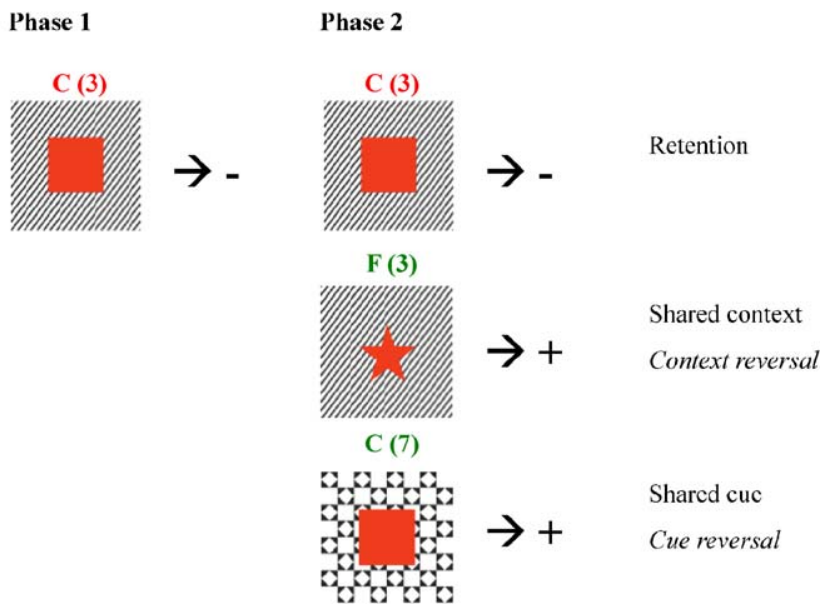

Fig. 1. Illustration of the cue and context reversal task. In cue change, new cards share the same context with the original card, but they have a new cue. In context change, new cards share the same cue with the original card, but the cue is presented in a new context. The task has two versions, one with black cues and color context (A) and the other with color cues and pattern context (B). Letters "A-G" refer to the eight cue shapes, and numbers " $1-8$ " refer to the eight contexts (colors and patterns). Green is assigned to positive outcome (+), red to negative outcome $(-)$ (see also Table 1). (For interpretation of the references to color in this figure legend, the reader is referred to the web version of this article.)

In order to investigate the role of MTL and PFC in cue and context reversal learning, we assessed a group of patient with newly diagnosed amnestic mild cognitive impairment (aMCI) and matched healthy controls. In contrast to patients with dementia, individuals with aMCI show relatively spared general cognitive abilities and daily functioning. However, they exhibit declarative memory impairments, which can be explained by MTL dysfunctions (Collie \& Maruff, 2000; Gauthier et al., 2006; Petersen et al., 1999; Shi, Liu, Zhou, Yu, \& Jiang, 2009; Whitwell et al., 2007). The majority of individuals with aMCI also exhibit executive dysfunctions, indicating the pathology of the PFC (Kramer et al., 2006; Price et al., 2010; Schmitter-Edgecombe \& Sanders, 2009). Beyond the fact that aMCI serves as a model condition for the impairment of multiple cognitive systems, which is, however, not entirely generalized in the early stage of the disorder, the context and cue reversal learning paradigm may provide an opportunity to develop new clinical testing tools.

We used neuropsychological tests sensitive to DLPFC, OFC/VMFC, and MTL functions in order to investigate the contri- 
Table 2

Demographic characteristics of the aMCI and control groups.

\begin{tabular}{lll}
\hline & aMCI $(n=30)$ & Control $(n=30)$ \\
\hline Age (years) & $60.2(6.2)$ & $61.0(6.8)$ \\
Male/female & $18 / 12$ & $18 / 12$ \\
Education (years) & $15.0(3.0)$ & $14.8(3.1)$ \\
Socioeconomic status (Hollingshead) & $39.5(8.5)$ & $38.6(9.3)$ \\
\hline
\end{tabular}

aMCI, amnestic mild cognitive impairment.

bution of these brain areas to cue and context reversal learning. Based on the pivotal role of the MTL in both context encoding and reversal, we hypothesized that the impairment of this brain region will be markedly associated with context reversal learning.

\section{Materials and methods}

\subsection{Participants}

Thirty individuals with aMCI and thirty matched healthy controls participated in the study (see Table 2 for a detailed description of the sample). The diagnosis of aMCI was established according to the Mayo Clinic Alzheimer's Disease Research Center criteria (Petersen et al., 1999). Exclusion criteria included neurological or psychiatric disorders, substance misuse, history of head trauma, vascular lesions on brain scans, and medications affecting central nervous system functions. Socioeconomic status was assessed using the Hollingshead Four Factor index (Hollingshead, 1975). Assessment was performed immediately after the establishment of clinical diagnosis in order to gain information from the earliest stage of aMCI and to avoid generalized cognitive impairment. The study was done in accordance with the Declaration of Helsinki. After a complete description of the study, a written informed consent was obtained from each participant.

\subsection{Neuropsychiatric Inventory}

The Neuropsychiatric Inventory is a semi-structured interview assessing the following behavioral anomalies: delusions, hallucinations, depression, anxiety, irritability/lability, aggression/agitation, euphoria, apathy, disinhibition, aberrant motor behavior, sleep disturbance, eating/appetite change, and stereotypical behavior (Cummings et al., 1994; Nyatsanza et al., 2003). Each behavioral domain is rated for severity (1-3) and frequency (1-4) (maximum score of 12 for each domain, maximum total score of 156$)$.

\subsection{Brain imaging}

We adopted the protocol of Myers et al. (2002, 2003) in order to verify MTL atrophy in patients with aMCI. In brief, each participant underwent a head magnetic resonance (MR) imaging (Siemens Trio 3T canner including a 3-D spoiled gradient recalled acquisition [SPGR] sequence). From the coronal SPGR scan, $3 \mathrm{~mm}$ axial reformats were produced parallel to the long axis of the hippocampus. In addition, coronal $1.3 \mathrm{~mm}$ reformats were produced perpendicular to the long axis of the hippocampus. Images were rated by two expert neuroradiologists blind to the diagnosis and to the aim of the study. We included aMCI patients only when both assessors revealed unequivocal bilateral MTL atrophy. Controls exhibited no atrophy.

The observer-based definition of MTL atrophy was verified by the quantitative volumetric measurement of the left and right hippocampi. We used the validated standard FreeSurfer image analysis pipeline (Martinos Center for Biomedical Imaging, Boston, MA, USA; http://surfer.nmr.mgh.harvard.edu/). Image processing included the removal of non-brain tissue with a hybrid watershed/surface deformation procedure, automated Talairach transformation, and segmentation of the subcortical white and grey matter (for methodological details, see Fischl et al. (2004) and Segonne et al. (2004))

\subsection{Cue and context reversal learning}

The task runs on a MacBook computer, programmed in SuperCard language. Participants play a card game in which they first see a pack of cards turned backward. For each card, they are asked to decide whether to flip it or to skip it. Participants need to flip the cards to reveal the pattern on it. Half of the cards predict reward (gaining points) and half predict punishment (losing points). The aim is to gain as many points as possible by flipping the reward cards and skipping the punishment cards.

The task has two phases. In the acquisition phase, four different cards are presented, ten times each (total of 40 trials). Each card contains a shape representing the cue and a background representing the context (Fig. 1). Two of the cards are associated with positive outcome (for example, card A1 with cue A and context 1 , Fig. $1 \mathrm{~A}$ and Table 1 ). If participants flip these cards, they gain 25 points. If they skip it, they neither gain nor lose points. The other two cards are associated with negative outcome (for example, card C3 with cue $C$ and context 3, Fig. 1B). If participants flip these cards, they lose 25 points, but if they skip it, they neither gain nor lose points.

The reversal phase is a direct continuation of the acquisition phase. On retention trials, the original cards are presented (e.g., cards A1 and C3, Fig. 1A/B) with the same outcome. On reversal trials, cards with a new context or a new cue are presented: (1) cards with a new cue but with the same context (e.g., card E1, Fig. 1A) or (2) cards with the same cue but with a new context (e.g., card A5, Fig. 1A). The outcome of these new cards is the opposite to that of the original cards. Therefore, if the original card A1 has a positive outcome in the acquisition phase, card E1 (new cue-shared context) and card A5 (shared cue-new context) will have a negative outcome in the reversal phase. Similarly, if the original card C3 has a negative outcome in the acquisition phase, cards $\mathrm{F} 3$ and $\mathrm{C} 7$ will have a positive outcome in the reversal phase (Fig. 1B and Table 1). Original and new cards are presented 10 times (total of 40 retention trials and 80 reversal trials).

To exclude the potential biasing effect of visual features of stimuli, two stimulus sets are used. In the first set, black cues are presented against a color background, whereas in the second set, red cues are presented against a black and white patterned background (Fig. 1A/B). Participants are randomly assigned to either stimulus sets The dependent measure is the percentage of correct decisions (flipping cards with positive outcome and skipping cards with negative outcome).

In summary, the task was designed to answer three questions: (1) Valance effect - what is the effect of positive vs. negative outcome on acquisition and reversal? Specifically, is it easier to acquire or reverse stimuli that are associated with negative or positive outcome? (2) Cue/context effect - how does reversal type (cue vs. context) affect performance? Specifically, are there differences in learning new contingencies for stimuli that share the same cue or same context? (3) Is there any interaction between valence and reversal type?

\subsection{Neuropsychological assessment}

All participants received the Rey Auditory Verbal Learning Test, Boston Naming Test, and semantic fluency test (Lezak, 1995). Deficits on these tests correlate with the subtle pathology of the temporal, parietal, and frontal lobe in aMCI (Nagy et al. 2007; Rose et al., 2006).

In addition to these tests, specific tasks sensitive to DLPFC, OFC/VMFC, and MTL functions were administered. DLPFC tasks included the extradimensional shift phase of the Cambridge Neuropsychological Test Automated Battery (CANTAB) (Owen, Roberts, Polkey, Sahakian, \& Robbins, 1991) and the One-touch Stockings of Cambridge (Owen et al., 1995).

In the CANTAB set-shifting task, participants perform a series of visual discriminations of stimuli consisting of shapes and lines. In the extra-dimensional shift phase new stimuli are introduced, and participants have to respond to the previously irrelevant dimension (shapes rather than lines). The dependent measure is the number of trials needed to meet criterion (6 consecutive correct responses) (Owen et al., 1991).

In the One-touch Stockings of Cambridge task(Owen et al., 1995), participants plan a series of sequential moves with different levels of complexity. During the test, two sets of three stockings are presented on a touch-screen, each set containing three colored balls. The task is to rearrange the balls in the bottom display so that the position of balls matches that in the top of the screen. Participants are requested to calculate the minimum number of moves to obtain the solution. The dependent measures are the mean proportion of correct solutions and the mean number of attempts for correct solution (Owen et al., 1995).

In addition to reversal learning, OFC/VMFC function was evaluated with the Holiday Apartment Task (Fellows, 2006; Meier, Charleston, \& Tippett, 2010). The task is to imagine a holiday for which participants should chose the best apartment. The apartments have different attributes (e.g., costs, noise level, neighborhood, and size), which are presented on the touch-screen in a table. Attributes are covered by white rectangles, which, once touched, disappear and the information for apartment attribute becomes visible. There are two trials: four apartments with six attributes and six apartments with seven attributes. The dependent measure is the search index, ranging from +1 to -1 : (movements across - movements down)/(movements across + movements down). If the search index is +1 , it means that all movements were across the table, searching by attribute. If the search index is -1 , it means that all movements were down, searching by apartment alternatives.

The CANTAB Paired Associates Learning test was used to assess MTL functions (Blackwell et al., 2004; Sahakian et al., 1988; Talpos, Winters, Dias, Saksida, \& Bussey 2009). During the short version of the test, 3 or 6 visual patterns are displayed in boxes placed in 6 different locations on a computer screen. The pattern is then presented in the centre of the display and the participant is asked to touch the box in which the pattern was previously seen. The task includes 10 presentations and recall phases if all patterns have not been placed correctly. The dependent measure is the number of errors in the 6-pattern phase, which is a sensitive measure of MTL pathology (Blackwell et al., 2004; Sahakian et al., 1988; Talpos et al., 2009).

\subsection{Data analysis}

The STATISTICA (version 9) software (StatSoft Inc., Tulsa, USA) was used for data analysis. All data were checked for normality of distribution using Kolmogorov-Smirnov tests. Repeated measures analyses of variance (ANOVAs) 
were used to compare cue and context reversal performance in controls and patients with aMCI. Scheffé's tests were used for post hoc comparisons. Neuropsychological test results and hippocampal volume were analyzed with two-tailed $t$ tests, together with the calculation of Cohen's effect size $(d)$. Pearson product-moment correlation coefficients were calculated to determine the relationship between cue and context reversal learning, neuropsychological test results, and hippocampal volume. The level of statistical significance was set at $\alpha<.05$.

\section{Results}

\subsection{Acquisition and retention of stimulus-outcome associations}

There were no significant differences between the two tasks (color vs. pattern context) $(F s<1, p s>.5)$, therefore data were collapsed and analyzed together.

We conducted a group (aMCI vs. controls) by outcome type (positive vs. negative feedback) by task phase (acquisition vs. retention) mixed model ANOVA on the percentage of correct responses. In this model, group was the between-subjects factor, and outcome type and task phase were the within-subjects factors. The ANOVA revealed no significant main effects of group and outcome type $(F s<3, p s>.1)$. The effect of task phase was significant $(F(1,58)=8.10, p=.006)$, indicating that participants performed better in the retention phase than in the acquisition phase. The ANOVA revealed no significant interactions among the factors $(F s<2, p>.2$ ) (Fig. 2).

\subsection{Cue and context reversal}

We conducted a group (aMCI vs. controls) by outcome type (positive vs. negative feedback) by reversal type (cue vs. context) mixed model ANOVA on the percentage of correct responses. In this model, group was the between-subjects factor and outcome and reversal types were the within-subjects factor. The ANOVA revealed significant main effects of group $(F(1,58)=38.21, p<.0001)$, outcome type $(F(1,58)=9.12, p=.004)$, and reversal type $(F(1,58)=51.94$, $p<.0001)$. Most importantly, we found a significant two-way inter- action between group and reversal type $(F(1,18)=27.93, p<.0001)$. Interactions between group and outcome type and among group, outcome type, and reversal type were not significant $(F s<3, p s>.1$ ).

Scheffé's tests conducted on the group by reversal type interaction indicated that aMCI patients were impaired on context reversal relative to controls $(p<.0001 ; d$ [Positive-to-Negative reversal] $=1.1 ; d$ [Negative-to-Positive reversal] $=1.4$ ), whereas they displayed normal performance on cue reversal $(p>.5 ; d$ [Positive-to-Negative reversal] $=.24 ; d$ [Negative-to-Positive reversal] =.31) (Fig. 3). In the aMCI group, performance was lower for context reversal relative to cue reversal $(p<.0001)$, whereas control participants performed similarly on cue and context reversal $(p>.5)$ (Fig. 3).

\subsection{Neuropsychological performance}

Table 3 depicts the comparison of aMCI patients and controls on neuropsychological tasks. Overall, aMCI patients exhibited marked deficits on declarative memory tests sensitive to MTL function and on some tasks related to DLPFC functions. Lexical and semantic processing was relatively less affected, and OFC/VMFC functions were spared.

Correlations between cue and context reversal and neuropsychological measures were modest. In controls, less efficient context reversal was associated with more errors on the paired associates test (Positive-to-Negative reversal: $r=-.64, p<.001$; Negative-toPositive reversal: $r=-.61, p<.001)$ and with lower performances on the Rey Auditory Verbal Learning test (Positive-to-Negative reversal: $r=.59, p=.001$; Negative-to-Positive reversal: $r=.40$, $p=.03$ ). In aMCI patients, the correlation between context reversal and paired associates learning was also significant (Positive-toNegative reversal: $r=-.61, p<.001$; Negative-to-Positive reversal: $r=-.63, p<.001)$. All other correlations between cue and context reversal learning and neuropsychological tests did not reach the level of statistical significance in either controls or patients $(-.40<r s<.40, p s>.05)$.
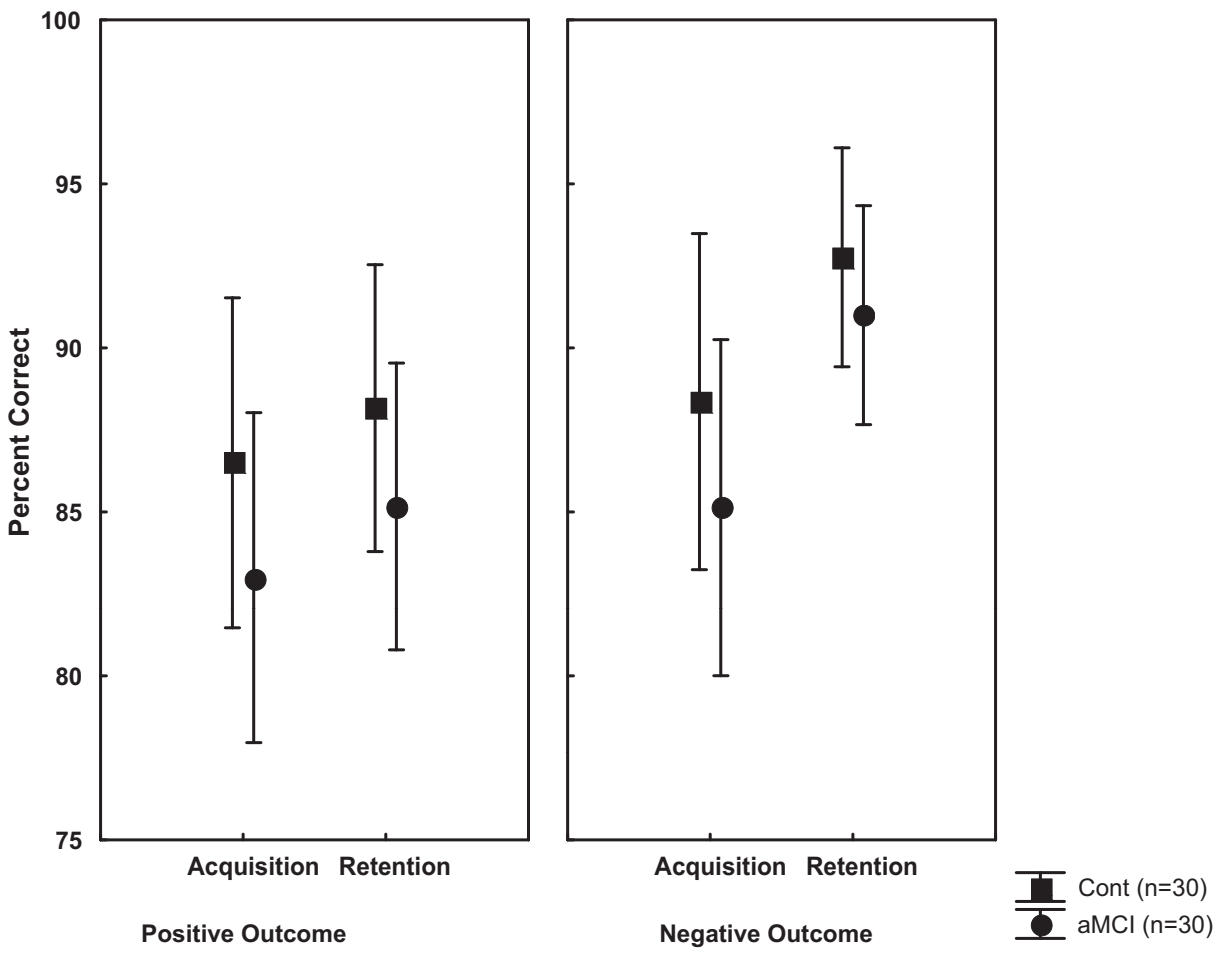

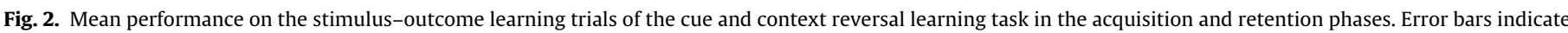
$95 \%$ confidence intervals. aMCI, amnestic mild cognitive impairment; Cont, controls. 

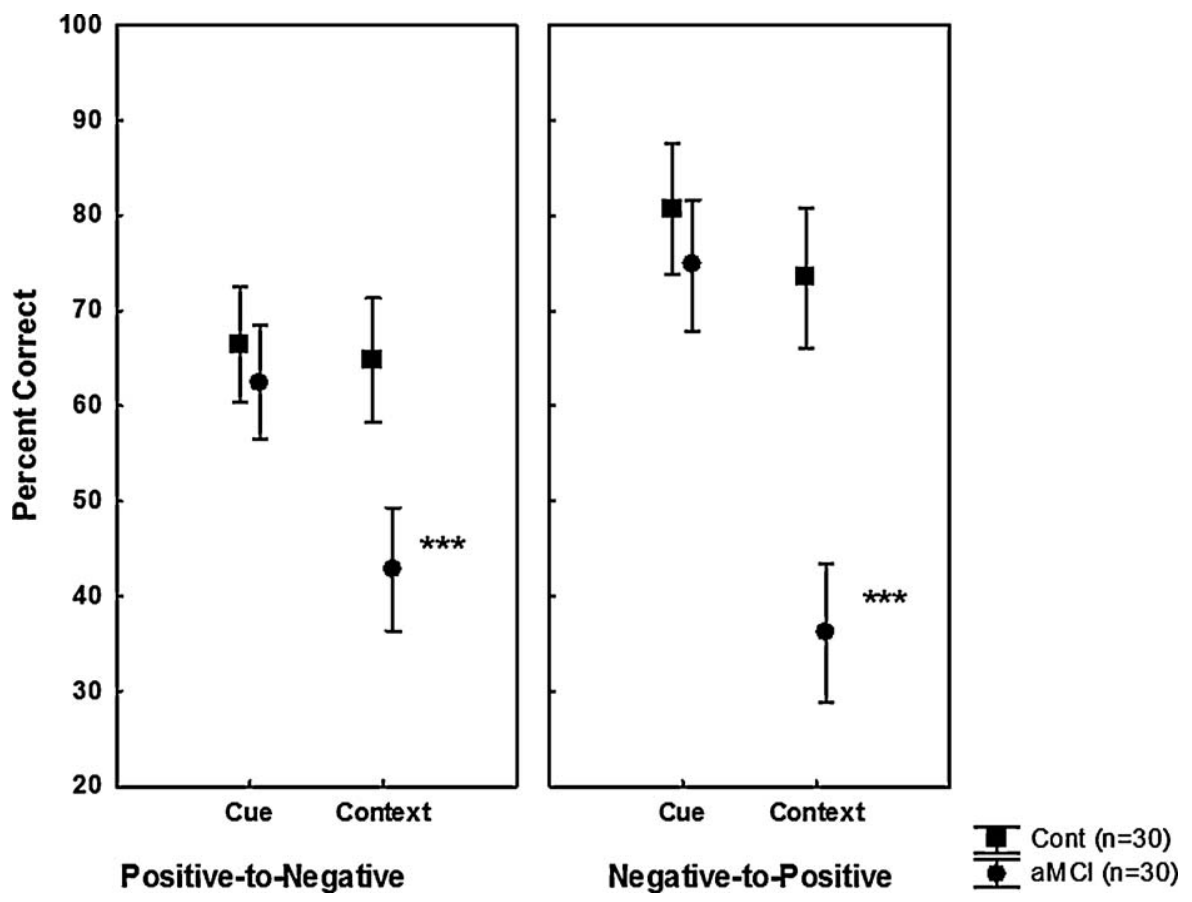

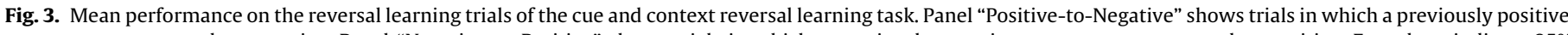

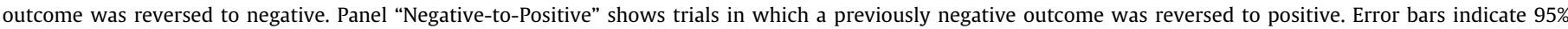
confidence intervals. aMCI, amnestic mild cognitive impairment; Cont, controls; ${ }^{* * *} p<.0001$, Scheffé's test.

Table 3

Performance by aMCI and control groups on neuropsychological tests.

\begin{tabular}{|c|c|c|c|c|c|c|}
\hline & Controls $(n=30)$ & $\operatorname{aMCI}(n=30)$ & $t$ & $d f$ & $p$ & $d$ \\
\hline Paired associates learning-6, mean number of errors & $4.6(2.9)$ & $13.4(4.6)$ & -8.79 & 58 & $<.0001$ & 1.5 \\
\hline Rey AVLT & $49.9(4.2)$ & $42.3(5.6)$ & 6.0 & 58 & $<.0001$ & 1.2 \\
\hline Boston Naming Test & $52.6(3.8)$ & $49.9(4.7)$ & 2.34 & 58 & .02 & .6 \\
\hline Semantic fluency & $19.5(4.1)$ & $17.3(4.2)$ & 2.10 & 58 & .04 & .5 \\
\hline Extradimensional shift & $17.7(6.8)$ & $20.9(5.7)$ & -2.13 & 58 & .04 & .5 \\
\hline OTS Cambridge, mean proportion of prefect solutions & $.69(.12)$ & $.56(.12)$ & 3.21 & 58 & .002 & 1.0 \\
\hline OTS Cambridge, mean number of attempts for perfect solution & $1.5(.7)$ & $2.2(1.3)$ & -2.34 & 58 & .02 & 6 \\
\hline Holiday Apartment Task, $4 \times 6$ (search index) & $.44(.35)$ & $.51(.31)$ & -.86 & 58 & .39 & .2 \\
\hline Holiday Apartment Task, $6 \times 7$ (search index) & $.51(.31)$ & $.66(.35)$ & -1.69 & 58 & .10 & .4 \\
\hline
\end{tabular}

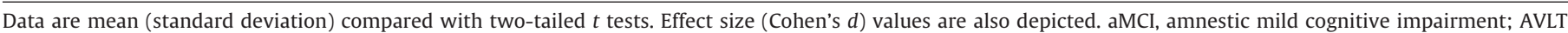
auditory verbal learning test; OTS, One-touch Stockings.

To further investigate the possibility that DLPFC dysfunctions contributed to impaired context reversal, we conducted a mediansplit analysis in which we compared context reversal learning in participants with higher and lower performances on CANTAB extradimensional shift and One-touch Stockings of Cambridge tasks. These analyses revealed that participants with higher and lower performances on tasks sensitive to DLPFC functions, as defined by the median-split analysis, performed similarly on context reversal $(F s<1, p s>.1$ ), which indicates that there was no effect of DLPFC function on context reversal.

\subsection{Neuropsychiatric Inventory}

Scores ranged from 0 to 8 . There were no aMCI patients who exhibited a behavioral domain in which clinically significant changes (score $>3$ ) was found.

\subsection{Hippocampal volume and test performance}

In accordance with the observer-based definition of MTL atrophy, results revealed significantly reduced hippocampal volumes in patients with aMCI relative to controls (left hip- pocampus, controls: $3418.3 \mathrm{~mm}^{3}(\mathrm{SD}=330.2)$, aMCI: $2340.7 \mathrm{~mm}^{3}$ $(\mathrm{SD}=462.8) ; t(58)=10.38, p<.0001, d=1.6$; right hippocampus, controls: $3456.1 \mathrm{~mm}^{3} \quad(\mathrm{SD}=325.9), \quad \mathrm{aMCI}=2383.7 \mathrm{~mm}^{3}$ $(\mathrm{SD}=495.3) ; t(58)=9.91, p<.0001, d=1.6)$.

In the control group, hippocampal volume did not correlate with test performances $(r s<.1)$. In the aMCI group, right hippocampal volume correlated with context reversal performance (Negative-to-Positive: $r=.42, p<.05$, Positive-to-Negative: $r=.37$, $p<.05)$. A similar relationship was found for paired associates learning $(r=-.40, p<.05)$. Other test results did not correlate with hippocampal volume $(r s<.2)$.

\section{Discussion}

The purpose of this study was to investigate cue and context reversal learning in aMCI and to assess its relationship with PFC and MTL functions. The most important finding was that cue and context reversal learning can be dissociated: patients with aMCl displayed intact cue reversal learning, but they showed significantly impaired context reversal learning. This finding raises the possibility that these types of learning are related to different neuronal mechanisms. Although patients with aMCI also exhibited 
executive deficits on tasks sensitive to DLPFC functions, context reversal learning was selectively associated with paired associates learning, which is a well-established neuropsychological marker of MTL dysfunctions in aMCI (Ahmed, Mitchell, Arnold, Nestor, \& Hodges, 2008; Blackwell et al., 2004; Talpos et al., 2009). This correlation was also observed in healthy controls. In addition, executive dysfunctions did not correlate with context reversal learning. These findings are consistent with results from recognition memory experiments showing that context effects are related to the MTL but not to the PFC (Vakil, Raz, \& Levy, 2010).

Using functional magnetic resonance imaging, de Rover et al. (2011) demonstrated differential hippocampal activation in MCI and control groups during the paired associates learning task: $\mathrm{MCI}$ patients displayed higher activation than controls at low memory loads and less at higher loads. Importantly, this functional impairment was confined to the hippocampal region, consistently with the structural alteration of it (grey matter reduction). Hanseeuw et al. (2011) showed that associative memory correlates with hippocampal volume in aMCI, which is similar to the results of Atienza et al. (2011) using a different paradigm. The fact that we found correlation between context reversal and paired associates learning, together with the evidence of MTL atrophy in our aMCI patients, suggest that context reversal learning is linked to the MTL. Furthermore, decreased right hippocampal volume was associated with less efficient context reversal learning.

Impaired context reversal learning in aMCI was independent of outcome valance, as it was observed both when previously negative outcomes became positive and when previously positive outcomes became negative. The valence-independence of context reversal learning dysfunction and spared cue reversal learning may indicate intact affective processing in our patients. Indeed, they showed normal performance on the Holiday Apartment Task, which is sensitive for VMFC/OFC function. Fellows (2006) demonstrated that patients with VMFC damage achieve a negative search index on this task, indicating an alternative-based rather than an attributebased acquisition strategy (organizing search around individual apartments instead of their attributes). In contrast, healthy participants and patients with lesion to the DLPFC exhibit attribute-based search, achieving a positive search index. The aMCI participants in this study showed a positive search index (Table 3), which is an evidence for intact VMFC function. Finally, the Neuropsychiatric Inventory did not reveal any behavioral changes that may indicate dysfunctional OFC/VMFC - amygdala system (Drevets, Price, \& Furey, 2008).

The most important limitation of the present study is the lack of voxel-based morphometry of the MTL. In addition, brain atrophy and dysfunctional neuronal activation are not confined to the MTL in aMCI (Hampstead, Stringer, Stilla, Amaraneni, \& Sathian, 2011; Nickl-Jockschat et al., 2011; Pihlajamäki, Jauhiainen, \& Soininen, 2009; Schroeter, Stein, Maslowski, \& Neumann, 2009), and therefore future studies should apply a whole-brain morphometry approach. However, our MRI protocol allows the detection of a robust and clinically relevant level of MTL atrophy, and in this way we were able to reduce the inherent heterogeneity of aMCI.

Beyond clinical implications, our results may also be relevant to delineate fine structure-function mapping in the MTL. For example, by the analysis of 210 neuropsychologically characterized individuals, Brickman, Stern, and Small (2010) demonstrated that delayed retention is associated with the basal metabolism of the entorhinal cortex, whereas recognition performance is associated with that of the dentate gyrus. Regarding episodic memory, it has been proposed that distinct items (e.g., objects and persons) are represented in the perirhinal and lateral entorhinal cortex, whereas spatial context of the item is processed in the parahippocampal and medial entorhinal cortex (Eichenbaum et al., 2007). According to the results of Mueller et al. (2010), the CA1-2 zone is better than the total hippocampal volume for discrimination between healthy controls and aMCI patients. As the hippocampus may be responsible for integration placing items in the right context (Eichenbaum et al., 2007), our paradigm is suitable for the investigation of item (cue)-context relationship in the framework of reinforcement and reversal learning.

Although the results of this study suggest a definitive specificity for the MTL, it is an oversimplification to imply that performance on a given task can be identified with the functioning of a specific brain region. In most cases, neuropsychological tasks cannot be "process pure" that they can index the functioning of a single circumscribed part of the brain. Although the context and cue reversal learning task was designed to be as "process pure" as possible, the inherent limitation of tasks regarding neuroanatomical specificity must be taken into consideration when our results are interpreted.

In conclusion, our results resolve some issues regarding the mechanism of reversal learning. We showed that reversal learning dysfunctions, specifically for context and not for cue, can occur when OFC/VMFC is spared and there is marked declarative memory impairment, most likely of MTL origin. Therefore, we propose that damage to the MTL selectively affects the reversal of contingencies related to the context, but not that related to the item. Using our task design, future studies will be able to delineate the interaction among the OFC/VMFC, fronto-striatal system, and MTL, which may play different roles in reinforcement and reversal learning.

\section{References}

Ahmed, S., Mitchell, J., Arnold, R., Nestor, P. J., \& Hodges, J. R. (2008). Predicting rapid clinical progression in amnestic mild cognitive impairment. Dementia and Geriatric Cognitive Disorders, 25, 170-177.

Atienza, M., Atalaia-Silva, K. C., Gonzalez-Escamilla, G., Gil-Neciga, E., SuarezGonzalez, A., \& Cantero, J. L. (2011). Associative memory deficits in mild cognitive impairment: The role of hippocampal formation. NeuroImage, 57, 1331-1342.

Birrell, J. M., \& Brown, V. J. (2000). Medial frontal cortex mediates perceptual attentional set shifting in the rat. Journal of Neuroscience, 20, 4320-4324.

Blackwell, A. D., Sahakian, B. J., Vesey, R., Semple, J. M., Robbins, T. W., \& Hodges, J. R. (2004). Detecting dementia: Novel neuropsychological markers of preclinical Alzheimer's disease. Dementia and Geriatric Cognitive Disorders, 17, 42-48.

Bouton, M. E. (1993). Context, time, and memory retrieval in the interferente paradigms of Pavlovian learning. Psychological Bulletin, 114, 80-99.

Brickman, A. M., Stern, Y., \& Small, S. A. (2010). Hippocampal subregions differentially associate with standardized memory tests. Hippocampus, doi:10.1002/hipo.20840 (Epub ahead of print)

Chudasama, Y., \& Robbins, T. W. (2006). Functions of frontostriatal systems in cognition: Comparative neuropsychopharmacological studies in rats, monkeys and humans. Biological Psychology, 73, 19-38

Collie, A., \& Maruff, P. (2000). The neuropsychology of preclinical Alzheimer's disease and mild cognitive impairment. Neuroscience and Biobehavioral Reviews, 24 , 365-374.

Cools, R., Clark, L., Owen, A. M., \& Robbins, T. W. (2002). Defining the neural mechanisms of probabilistic reversal learning using event-related functional magnetic resonance imaging. Journal of Neuroscience, 22, 4563-4567.

Cummings, J. L., Mega, M., Gray, K., Rosenberg-Thompson, S., Carusi, D. A., \& Gornbein, J. (1994). The neuropsychiatric inventory: Comprehensive assessment of psychopathology in dementia. Neurology, 44, 2308-2314.

de Rover, M., Pironti, V. A., McCabe, J. A., Acosta-Cabronero, J., Arana, F. S., MoreinZamir, S., et al. (2011). Hippocampal dysfunction in patients with mild cognitive impairment: A functional neuroimaging study of a visuospatial paired associates learning task. Neuropsychologia, 49, 2060-2070.

Dias, R., Robbins, T. W., \& Roberts, A. C. (1996). Dissociation in prefrontal cortex of affective and attentional shifts. Nature, 380, 69-72.

Drevets, W. C., Price, J. L., \& Furey, M. L. (2008). Brain structural and functional abnormalities in mood disorders: Implications for neurocircuitry models of depression. Brain Structure and Function, 213, 93-118.

Eichenbaum, H., Yonelinas, A. R., \& Ranganath, C. (2007). The medial temporal lobe and recognition memory. Annual Review of Neuroscience, 20, 123-152.

Fellows, L. K. (2006). Deciding how to decide: Ventromedial frontal lobe damage affects information acquisition in multi-attribute decision making. Brain, 129, 944-952.

Fischl, B., Salat, D. H., van der Kouwe, A. J., Makris, N., Ségonne, F., Quinn, B. T., et al. (2004). Sequence-independent segmentation of magnetic resonance images. NeuroImage, 23(Suppl. 1), S69-S84.

Frank, M. J., \& Claus, E. D. (2006). Anatomy of a decision: Striato-orbitofrontal interactions in reinforcement learning, decision making, and reversal. Psychological Review, 113, 300-326. 
Gauthier, S., Reisberg, B., Zaudig, M., Petersen, R. C., Ritchie, K., Broich, K., et al. (2006). Mild cognitive impairment. Lancet, 367, 1262-1270.

Gluck, M. A., \& Myers, C. E. (2001). Gateway to memory: An introduction to neural network modeling of the hippocampus in learning and memory. Cambridge, MA: MIT Press.

Good, M., \& Honey, R. C. (1991). Conditioning and contextual retrieval in hippocampal rats. Behavioral Neuroscience, 105, 499-509.

Hampstead, B. M., Stringer, A. Y., Stilla, R. F., Amaraneni, A., \& Sathian, K. (2011). Where did I put that? Patients with amnestic mild cognitive impairment demonstrate widespread reductions in activity during the encoding of ecologically relevant object-location associations. Neuropsychologia, 49, 23492361.

Hanseeuw, B., Dricot, L., Kavec, M., Grandin, C., Seron, X., \& Ivanoiu, A. (2011). Associative encoding deficits in amnestic mild cognitive impairment: A volumetric and functional MRI study. NeuroImage, 56, 1743-1748.

Hollingshead, A. B. (1975). Four factor index of social status. Unpublished manuscript, Yale University, New Haven, CT.

Kramer, J. H., Nelson, A., Johnson, J. K., Yaffe, K., Glenn, S., Rosen, H. J., et al. (2006). Multiple cognitive deficits in amnestic mild cognitive impairment. Dementia and Geriatric Cognitive Disorders, 22, 306-311.

Lezak, M. (1995). Neuropsychological assessment. Oxford: Oxford University Press.

Marston, H. M., Everitt, B. J., \& Robbins, T. W. (1993). Comparative effects of excitotoxic lesions of the hippocampus and septum/diagonal band on conditional visual discrimination and spatial learning. Neuropsychologia, 31, 10991118

Mayes, A. R., Macdonald, C., Donlan, L., Pears, J., \& Meudell, P. R. (1992). Amnesics have a disproportionately severe memory deficit for interactive context. Quarterly Journal of Experimental Psychology, 45, 265-297.

Meier, S. L., Charleston, A. J., \& Tippett, L. J. (2010). Cognitive and behavioural deficits associated with the orbitomedial prefrontal cortex in amyotrophic lateral sclerosis. Brain, 133, 3444-3457.

Mueller, S. G., Schuff, N., Yaffe, K., Madison, C., Miller, B., \& Weiner, M. W. (2010). Hippocampal atrophy patterns in mild cognitive impairment and Alzheimer's disease. Human Brain Mapping, 31, 1339-1347.

Myers, C. E., Deluca, J., Hopkins, R. O., \& Gluck, M. A. (2006). Conditional discrimination and reversal in amnesia subsequent to hypoxic brain injury or anterior communicating artery aneurysm rupture. Neuropsychologia, 44, 130-139.

Myers, C. E., \& Gluck, M. A. (1994). Context, conditioning, and hippocampal representation in animal learning. Behavioral Neuroscience, 108, 835-847.

Myers, C. E., Kluger, A., Golomb, J., Ferris, S., de Leon, M., Schnirman, G., et al. (2002). Hippocampal atrophy disrupts transfer generalization in non-demented elderly. Journal of Geriatric Psychiatry and Neurology, 15, 82-90.

Myers, C. E., Shohamy, D., Gluck, M. A., Grossman, S., Kluger, A., Ferris, S., et al. (2003). Dissociating hippocampal versus basal ganglia contributions to learning and transfer. Journal of Cognitive Neuroscience, 15, 185-193.

Nagy, H., Kéri, S., Myers, C. E., Benedek, G., Shohamy, D., \& Gluck, M. A. (2007). Cognitive sequence learning in Parkinson's disease and amnestic mild cognitive impairment: Dissociation between sequential and non-sequential learning of associations. Neuropsychologia, 45, 1386-1392.

Nickl-Jockschat, T., Kleiman, A., Schulz, J. B., Schneider, F., Laird, A. R., Fox, P. T., et al. (2011). Neuroanatomic changes and their association with cognitive decline in mild cognitive impairment: A meta-analysis. Brain Structure and Function, doi:10.1007/s00429-011-0333-x (Epub ahead of print)

Nyatsanza, S., Shetty, T., Gregory, C., Lough, S., Dawson, K., \& Hodges, J. R. (2003). A study of sterotypic behaviours in Alzheimer's disease and frontal and temporal variant frontotemporal dementia. Journal of Neurology, Neurosurgery and Psychiatry, 74, 1398-1402.

Owen, A. M., Roberts, A. C., Polkey, C. E., Sahakian, B. J., \& Robbins, T. W. (1991). Extra-dimensional versus intra-dimensional set shifting performance following frontal lobe excisions, temporal lobe excisions or amygdalohippocampectomy in man. Neuropsychologia, 29, 993-1006.
Owen, A. M., Sahakian, B. J., Hodges, J. R., Summers, B. A., Polkey, C. E., \& Robbins, T. W. (1995). Dopamine-dependent frontostriatal planning deficits in early Parkinson's disease. Neuropsychology, 9, 126-140.

Penick, S., \& Solomon, P. R. (1991). Hippocampus, context, and conditioning. Behavioral Neuroscience, 105, 611-617.

Petersen, R. C., Smith, G. E., Waring, S. C., Ivnik, R. J., Tangalos, E. G., \& Kokmen, E. (1999). Mild cognitive impairment: Clinical characterization and outcome. Archives of Neurology, 56, 303-308.

Pihlajamäki, M., Jauhiainen, A. M., \& Soininen, H. (2009). Structural and functiona MRI in mild cognitive impairment. Current Alzheimer Research, 6, 179-185.

Price, S. E., Kinsella, G. J., Ong, B., Mullaly, E., Phillips, M., Pangnadasa-Fox, L., et al. (2010). Learning and memory in amnestic mild cognitive impairment: Contribution of working memory. Journal of the International Neuropsychological Society $16,342-351$.

Robbins, T. W., \& Arnsten, A. F. (2009). The neuropsychopharmacology of frontoexecutive function: Monoaminergic modulation. Annual Review of Neuroscience. 32, 267-287.

Rose, S. E., McMahon, K. L., Janke, A. L., O’Dowd, B., de Zubicaray, G., Strudwick, M. W., et al. (2006). Diffusion indices on magnetic resonance imaging and neuropsychological performance in amnestic mild cognitive impairment. Journal of Neurology, Neurosurgery and Psychiatry, 77, 1122-1128.

Sahakian, B. J., Morris, R. G., Evenden, J. L., Heald, A., Levy, R., Philpot, M., et al. (1988) A comparative study of visuospatial memory and learning in Alzheimer-type dementia and Parkinson's disease. Brain, 111, 695-718.

Schmitter-Edgecombe, M., \& Sanders, C. (2009). Task switching in mild cognitive impairment: Switch and nonswitch costs. Journal of the International Neuropsychological Society, 15, 103-111.

Schroeter, M. L., Stein, T., Maslowski, N., \& Neumann, J. (2009). Neural correlates of Alzheimer's disease and mild cognitive impairment: A systematic and quantitative meta-analysis involving 1351 patients. Neurolmage, 47, 1196-1206.

Segonne, F., Dale, A. M., Busa, E., Glessner, M., Salat, D., Hahn, H. K., et al. (2004) A hybrid approach to the skull stripping problem in MRI. NeuroImage, 22, 1060-1075.

Shi, F., Liu, B., Zhou, Y., Yu, C., \& Jiang, T. (2009). Hippocampal volume and asymmetry in mild cognitive impairment and Alzheimer's disease: Meta-analyses of MRI studies. Hippocampus, 19, 1055-1064.

Shohamy, D., Myers, C. E., Hopkins, R. O., Sage, J., \& Gluck, M. A. (2009). Distinct hippocampal and basal ganglia contributions to probabilistic learning and reversal. Journal of Cognitive Neuroscience, 21, 1821-1833.

Swainson, R., Rogers, R. D., Sahakian, B. J., Summers, B. A., Polkey, C. E., \& Robbins T. W. (2000). Probabilistic learning and reversal deficits in patients with Parkinson's disease or frontal or temporal lobe lesions: Possible adverse effects of dopaminergic medication. Neuropsychologia, 38, 596-612.

Talpos, J. C., Winters, B. D., Dias, R., Saksida, L. M., \& Bussey, T. J. (2009). A nove touchscreen-automated paired-associate learning (PAL) task sensitive to pharmacological manipulation of the hippocampus: A translational rodent mode of cognitive impairments in neurodegenerative disease. Psychopharmacology (Berl), 205, 157-168.

Vakil, E., Raz, T., \& Levy, D. A. (2007). Multifactorial context effects on visual recognition memory. Quarterly Journal of Experimental Psychology, 60, 916-923.

Vakil, E., Raz, T., \& Levy, D. A. (2010). Probing the brain substrates of cognitive processes responsible for context effects on recognition memory. Aging, Neuropsychology, and Cognition, 17, 519-544.

Whitwell, J. L., Przybelski, S. A., Weigand, S. D., Knopman, D. S., Boeve, B. F., Petersen R. C., et al. (2007). 3D maps from multiple MRI illustrate changing atrophy patterns as subjects progress from mild cognitive impairment to Alzheimer's disease. Brain, 130, 1777-1786.

Wickens, D. D. (1987). Memory and learning: The Ebbinghaus centennial conference In D. S. Gorfein, \& R. R. Hoffman (Eds.), The dual meanings of context: Implications for research, theory and applications (pp. 135-152). Hillsdale, NJ: Lawrence Erlbaum Associates, Inc. 